\title{
Indications of esophageal cancer for endoscopic submucosal dissection, curability, and future perspectives
}

\author{
Ryu Ishihara \\ Department of Gastrointestinal Oncology, Osaka International Cancer Institute, Osaka 541-8567, Japan. \\ Correspondence to: Dr. Ryu Ishihara, Department of Gastrointestinal Oncology, Osaka International Cancer Institute, 3-1-69 \\ Otemae, Chuo-ku, Osaka 541-8567, Japan. E-mail: ryu1486@gmail.com
}

How to cite this article: Ishihara R. Indications of esophageal cancer for endoscopic submucosal dissection, curability, and future perspectives. Mini-invasive Surg 2021;5:36. https://dx.doi.org/10.20517/2574-1225.2021.72

Received: 1 Jun 2021 First Decision: 21 Jun 2021 Revised: 27 Jun 2021 Accepted: 7 Jul 2021 First online: 8 Jul 2021

Academic Editor: Shinji Tanaka Copy Editor: Yue-Yue Zhang Production Editor: Yue-Yue Zhang

\begin{abstract}
This review considers the preferred preoperative examinations, indications for endoscopic submucosal dissection (ESD), and curative ability of ESD in patients with esophageal squamous cell carcinoma (SCC). Endoscopic evaluation by non-magnifying endoscopy followed by magnifying endoscopy is a common procedure for diagnosing invasion depth of superficial esophageal SCCs in Japan. However, endoscopic ultrasonography may increase overdiagnosis of the depth of cancer invasion, and therefore should not be performed routinely. Image-enhanced magnifying endoscopy or iodine staining is recommended for diagnosing the lateral extent of esophageal SCC. The indications for ESD include clinical T1a-epithelial/lamina propria (EP/LPM) NOMO non-circumferential lesions, clinical T1a EP/LPM NOMO circumferential lesions $\leq 50 \mathrm{~mm}$, and clinical T1a-muscularis mucosae/T1b-submucosa 1 cancer (invading submucosa by $\leq 200 \mu \mathrm{m}$ ) NOMO non-circumferential lesions. Pathological T1a EP/LPM without vascular invasion is defined as curative resection, while pathological T1a MM without vascular invasion is considered as non-curative resection, with undetermined recommendations for additional treatment. Pathological T1b cancer invading the submucosa or pathological vascular invasion-positivity is considered as non-curative resection, and additional treatment is recommended. An accurate preoperative diagnosis, appropriate indication, and adequate curability assessment based on the pathological diagnosis of resected specimens are important for effective ESD.
\end{abstract}

Keywords: Esophageal cancer, cancer invasion depth, endoscopic submucosal dissection 


\section{INTRODUCTION}

Esophageal cancer is the seventh most common cancer and the sixth most common cause of cancer-related death worldwide, with 572,000 new cases and 509,000 deaths in $2018^{[1]}$. Although the incidence of esophageal adenocarcinoma is increasing rapidly in Europe and North America, esophageal squamous cell carcinoma (SCC) remains the most common histological type, accounting for $80 \%$ of all esophageal cancers worldwide ${ }^{[1]}$.

The overall survival of patients with advanced esophageal cancer remains poor, regardless of histological type. However, when diagnosed at an early stage, esophageal cancer can be cured by endoscopic submucosal dissection (ESD), surgical resection, or chemoradiotherapy. Superficial SCC is defined as cancer limited to the mucosa or the submucosa. The treatment strategy for superficial SCC of the esophagus is determined based on the preoperative diagnosis of cancer invasion depth, lateral extent of the cancer, and metastasis. The curative ability of tumor resection is usually determined by the histologic findings of the resected specimen. This review will discuss the preferred preoperative examinations, indications for ESD, and curative ability of ESD in patients with esophageal SCC.

\section{PREOPERATIVE EXAMINATIONS}

\section{Diagnosis of cancer invasion depth}

Endoscopic evaluation by non-magnifying endoscopy (non-ME) followed by magnifying endoscopy (ME) is the common procedure for diagnosing invasion depth of superficial esophageal SCC in Japan. Endoscopic ultrasonography (EUS) is also used to diagnose cancer invasion depth but is currently not used as a standard procedure because of conflicting results regarding its diagnostic accuracy ${ }^{[2,3]}$. Although EUS is recommended for staging T1 esophageal cancer in some guidelines ${ }^{[4-6]}$ and by some experts ${ }^{[7]}$, it is not recommended in other guideline ${ }^{[8,9]}$. A recent multicenter study was conducted to evaluate the additional diagnostic value of EUS following non-ME+ME for differentiating superficial SCC into M/SM1 cancer (mucosal cancer/cancer invading into the submucosa by $\leq 200 \mu \mathrm{m}$ ) and $\geq \mathrm{SM} 2$ cancer (cancer invading into the submucosa $>200 \mu \mathrm{m})^{[10]}$. Additional use of EUS after non-ME+ME increased the proportion of overdiagnoses by $6.6 \%(21.6 \%$ vs. $28.2 \%$, one-sided $P=0.93)$, with similarly increased tendencies for overdiagnosis in all subgroup analyses. Although the addition of EUS reduced the proportion of underdiagnoses by $4.5 \%$ (29.2\% vs. $24.7 \%)$, it did not improve the accuracy of distinguishing between M/SM1 and $\geq$ SM2 superficial SCCs. Overdiagnosis of the depth of invasion means that cancers potentially curable by endoscopic resection may be treated by esophagectomy, while underdiagnosed cancers may be treated by endoscopic resection, with no curative effect. An increase in overdiagnosis is considered to have a greater impact than underdiagnosis, because over diagnosed patients may receive unnecessary esophagectomy, which is more invasive than unnecessary endoscopic resection caused by an underdiagnosis. Similar results were reported in other studies evaluating the usefulness of additional EUS $^{[1,1,12]}$. Considering the risk-benefit balance of adding EUS, the current results suggest that EUS should not be performed routinely in patients with superficial esophageal SCC.

\section{Diagnosis of lateral extent}

The Esophageal Cancer Practice Guidelines 2017 $7^{[4]}$ suggest that the extent of endoscopic resection is closely related to the risk of stenosis, and it is therefore "strongly recommended to evaluate the circumferential extent of the lesion preoperatively". Image-enhanced magnifying endoscopy or iodine staining is recommended to diagnose the lateral extent of the lesion [Figure 1], with the latter allowing clear delineation of the lesion border. However, use of high concentrations of iodine solution may cause the superficial epithelium to peel off, making a subsequent diagnosis difficult, and thus iodine solution should be used at a low concentration of $\leq 1 \%$. 


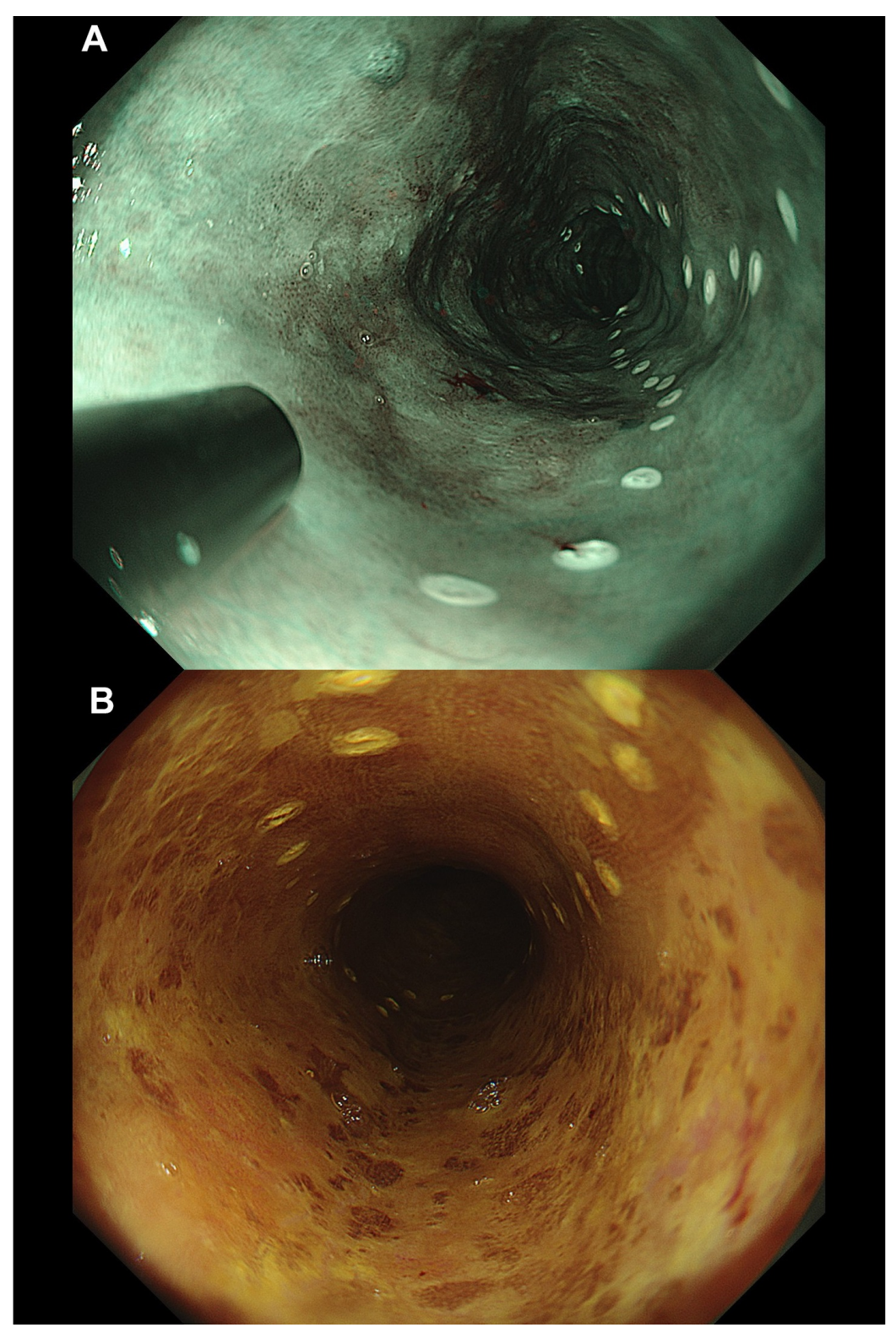

Figure 1. Diagnosis of lateral extent. (A) The lateral extent of the cancer was initially diagnosed using narrow-band imaging. (B) The lateral extent was then confirmed by iodine staining.

\section{INDICATIONS FOR ESD [Table 1]}

\section{Cancer invasion depth}

The indication for ESD in patients with T1NoMo esophageal SCC is determined mainly based on cancer invasion depth and the lateral extent of the cancer. Clinically diagnosed (c)T1a-epithelial/lamina propria (EP/LPM) cancers are considered good candidates for ESD and are thus regarded as an indication for ESD. The committee for the Japanese ESD/endoscopic mucosal resection (EMR) Guidelines ${ }^{[13]}$ previously discussed the validity of T1a-muscularis mucosae/T1b-submucosa 1 (MM/SM1) as an indication for ESD. However, there is considerable discrepancy between (c)MM/SM1 and pathologically diagnosed (p)MM/SM1, and these should thus be treated separately. The above committee discussed the validity of (c)MM/SM1 as an indication for ESD. 
$\sqrt{ }$ Clinical T1a-epithelial/lamina propria (EP/LPM) NOMO non-circumferential lesion

$\sqrt{ }$ Clinical T1a EP/LPM NOMO circumferential lesion $\leq 50 \mathrm{~mm}$

$\sqrt{ }$ Clinical T1a MM/T1b SM1 cancer (invading submucosa by $\leq 200 \mu \mathrm{m}$ ) NOMO non-circumferential lesion

T1a MM/T1b SM1: T1a-Muscularis Mucosae/T1b-Submucosa 1.

Previous reports ${ }^{[14-18]}$ showed that $27.4 \%-55.2 \%$ of cancers diagnosed as (c)MM/SM1 before treatment were (p)EP/LPM cancers [Figure 2], for which endoscopic resection is highly likely to be curative. This indicates that the accuracy of preoperative diagnosis for (c)MM/SM1 cancers is poor, and that a considerable proportion of esophageal SCC, which is curable by ESD, is included in (c)MM/SM1 cancers. Based on these facts, (c)MM/SM1 cancers are considered as an indication for ESD.

\section{Lateral extent of cancer}

Although ESD is an effective treatment, extensive esophageal endoscopic resection can lead to postoperative esophageal strictures, with rates of postoperative stricture after non-circumferential and whole circumferential resection of $60.7 \%-75 \%$ and $100 \%$, respectively, if preventive measures are not applied ${ }^{[19-21]}$. Stricture after esophageal ESD causes dysphagia and requires multiple, long-term endoscopic balloon dilatations. It thus has a negative impact on the patient's quality of life and may delay additional chemoradiotherapy after non-curative resection. However, the use of appropriate preventive measures can reduce the proportion of strictures after non-circumferential resection to $11.3 \%-36.2 \%^{[19,20,22]}$. Noncircumferential lesions are thus considered as an indication for ESD, whereas the risk of stricture following circumferential resection remains high, despite preventive measures.

The application of stenosis-preventive measures following circumferential resection was associated with stenosis rates of $76 \%$ in 45 patients who received steroid injection therapy ${ }^{[23-27]}, 55 \%$ in 44 patients who received oral steroid therapy ${ }^{[25,26,28-30]}$, and $71 \%$ in 14 patients who received both injected and oral steroid therapy ${ }^{[19]}$. However, these studies included widespread lesions with a mean major axis length of $6 \mathrm{~cm}$. A previous report ${ }^{[26]}$ showed that a resection diameter $>50 \mathrm{~mm}$ increased the stricture risk: when the major resection axis length was $>50 \mathrm{~mm}, 85 \%$ of patients (11/13 patients) required at least six sessions of dilatations, compared with only $17 \%$ of patients (1/6 patients) with a length $\leq 50 \mathrm{~mm}$. Furthermore, the administration of oral steroid prednisolone at a starting dose of $30 \mathrm{mg}$ and tapered for 12-18 weeks limited the stenosis rate to $27.3 \%$ ( $3 / 11$ patients) in patients who underwent whole-circumferential resection, requiring a mean of only 1.6 sessions of balloon dilatation ${ }^{[25]}$. These reports confirm that stricture relief can be achieved more easily in tumors with a major axis length $\leq 50 \mathrm{~mm}$, and effective methods are being developed to prevent stricture following whole-circumferential stenosis.

Expected curability is another important factor determining the indication for ESD. Although, no studies have reported on the pathologic results for CEP/LPM cancer with circumferential extent, a previous report showed that approximately $70 \%$ of cEP/LPM cancers $\geq 50 \mathrm{~mm}$ were (p)EP/LPM cancers ${ }^{[3]}$. Conversely, however, another study ${ }^{[32]}$ showed that only $14 \%$ (2/14 lesions) of (c)MM/SM1 whole-circumferential cancers were (p)EP/LPM cancers. In addition, 86\% (12/14 lesions) of (c)MM/SM1 whole-circumferential cancers were at high risk of metastasis (submucosal cancer or vascular invasion positive) or had lymph node metastasis. From the perspective of accuracy for preoperative diagnosis of cancer invasion depth, further investigation is needed regarding the adequacy of ESD for (c)MM/SM1 whole-circumferential cancers. Considering the expectancy of curability and postoperative complications, ESD is therefore recommended for CT1a-EP/LPM superficial SCCs with a major axis length $\leq 50 \mathrm{~mm}$ and involving the entire circumference of the esophagus, upon implementing preventive measures for stenosis. 


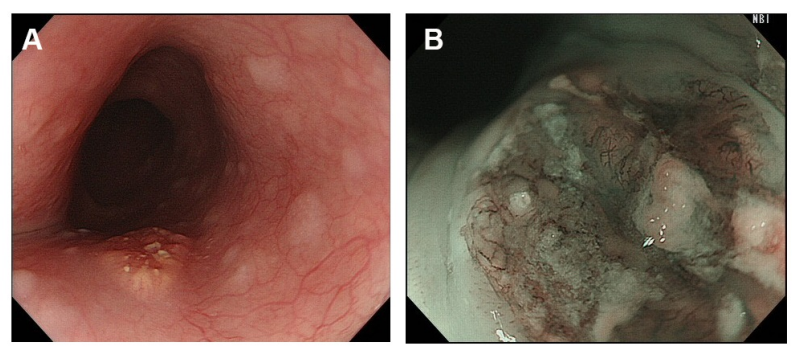

C

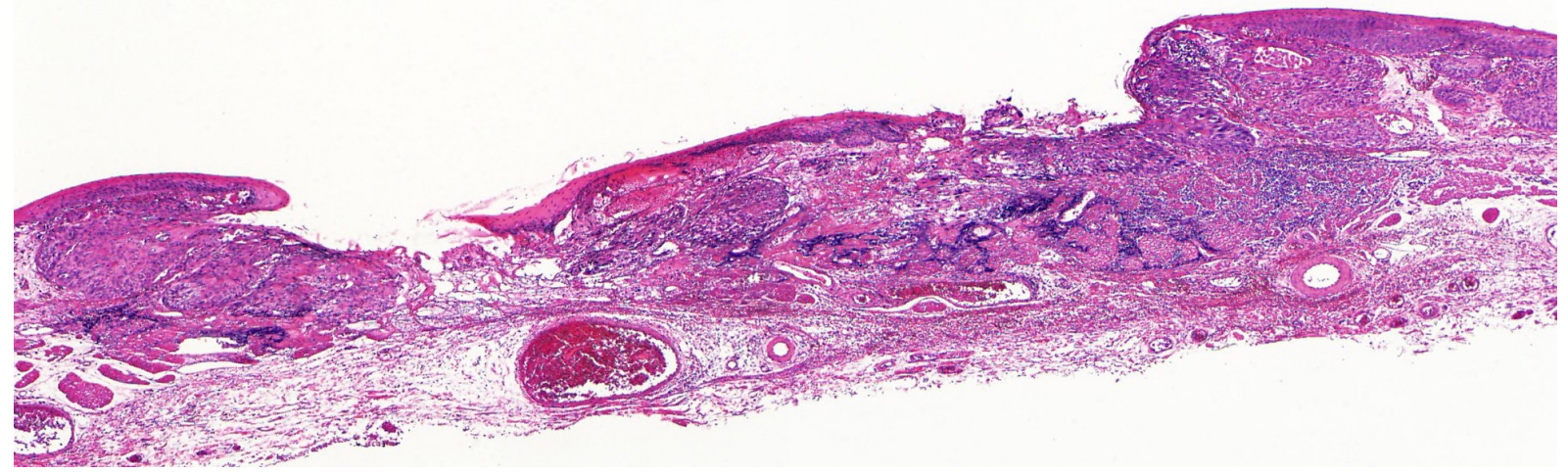

Figure 2. Clinical T1a-muscularis mucosae/T1b-submucosa 1 (MM/SM1) cancer finally diagnosed as pathological lamina propria cancer by pathological examination of resected specimen. (A) Lesion was slightly elevated on white light imaging. (B) Lesion was mainly occupied by B2 vessels. (C) Pathological diagnosis of resected specimen in our facility was cancer invading lamina propria without vascular invasion (hematoxylin and eosin staining)

\section{Diagnosis of metastasis}

To determine treatment strategy for T1 esophageal SCC, clinical stage of the cancer should be confirmed by such means as computed tomography of the neck, chest, and abdomen, and positron-emission tomography. Although ESD is indicated to patients with T1NoMo, accuracy of clinical diagnosis for NoMo is not yet elucidated. A previous study showed that the accuracy of clinical No for T1b cancer was $73 \%{ }^{[33]}$. In other words, despite a clinical diagnosis of No, there still exists a risk of metastasis. We therefore have to conduct strict curability assessment after ESD and apply additional treatment to patients with high risk for metastasis even when the cancer is clinical No.

\section{CURABILITY ASSESSMENT [Table 2]}

Curability following ESD is determined based on the histological findings of the resected specimens. Horizontal and vertical margin statuses correlate well with the risk of local recurrence, while cancer invasion depth and vascular invasion statuses correlate well with the risk of metastasis. For the assessment of vascular invasion, additional assessment using immunostaining need to be considered because it may enhance the detection of vascular invasion ${ }^{[34]}$.

\section{Horizontal and vertical margin status}

The resection can be judged as curative in terms of margin status if both the horizontal and vertical margins are negative. If the horizontal margin is positive, careful surveillance is recommended because of the risk of local recurrence, and if the vertical margin is positive, additional treatment, such as esophagectomy or chemoradiotherapy, is recommended to eradicate the residual cancer. 
Table 2. Curative ability assessment of endoscopic submucosal dissection based on cancer invasion depth and vascular invasion status

Curative resection

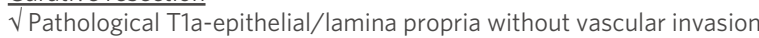

Non-curative resection and undetermined recommendation for additional treatment

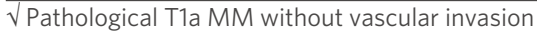

Non-curative resection and additional treatment recommended

$\sqrt{\text { Pathological T1b cancer invading the submucosa }}$

$\sqrt{ }$ Pathological vascular invasion-positive

MM: Muscularis mucosae.

\section{Cancer invasion depth and vascular invasion}

(p)EP/LPM cancer

Although metastasis may develop in (p)EP/LPM cancers without vascular invasion, the risk of the metastasis is as low as $0.4 \%{ }^{[35]}$. The resection is judged as curative if the histological findings of the resected specimen show (p)EP/LPM cancer without vascular invasion and if the horizontal and vertical margins are negative. Other cancers, (p)MM, (p)SM, or tumors with vascular invasion are considered as non-curable in terms of cancer invasion depth and vascular invasion.

\section{(p)MM cancer}

The ESD/EMR Guidelines for Esophageal Cancer ${ }^{[13]}$ analyzed the incidences of metastasis of (p)MM cancers in surgically and endoscopically resected patients. For (p)MM cancer without vascular invasion, the lymph node metastasis rate was $4 / 38(10.5 \%)$ in surgically resected patients ${ }^{[36]}$, compared with $12 / 216(5.6 \%)$ in the follow-up observation group after endoscopic resection ${ }^{[13]}$. Given the considerable risk of metastasis, pathologically diagnosed (p)MM cancer without vascular invasion is judged as non-curable. However, considering the reduced quality of life and the possibility of treatment-related death associated with additional surgical resection, as well as delayed adverse events and treatment-related deaths following additional chemoradiotherapy, these treatments are not conducted in most cases. In addition, patients are usually informed that metastasis can occur at certain rates and that it is crucial to perform careful follow-up, including screening for metastasis.

Regarding (p)MM cancer with vascular invasion, the lymph node metastasis rate was 5/12 (41.7\%) in surgically resected patients ${ }^{[36]}$ compared with $3 / 14$ patients $(21.4 \%)$ in the follow-up observation group after endoscopic resection ${ }^{[13]}$. Given the high risk of metastasis, pathologically diagnosed (p)MM cancer with vascular invasion is thus considered to be non-curable, but additional treatment with surgical resection or chemoradiotherapy is recommended for these patients.

\section{(p)SM cancer}

The ESD/EMR Guidelines for Esophageal Cancer analyzed the incidences of metastasis of (p)SM cancers in surgically and endoscopically resected patients. Analyses of resected specimens from patients with (p)SM1/SM2 esophageal SCC who received surgical resection as first-line treatment, including patients with vascular invasion, showed concurrent lymph node metastasis in $43 / 170$ patients (25.3\%) with (p)SM1 cancers and 49/196 patients (25\%) with (p)SM2 cancers, compared with 8/43 patients (18.6\%) with (p)SM1 cancers and $3 / 20$ patients (15\%) with (p)SM2 cancers in the follow-up observation group of patients who received endoscopic resection as first-line treatment, including patients with vascular invasion. In addition, recent retrospective study showed that lymphatic invasion and (p)SM2 were independent risk factors for metastatic recurrence ${ }^{[37]}$. Given the high proportion of metastasis, a histological finding of (p)SM cancer is judged as non-curable, and additional treatment with surgical resection or chemoradiotherapy is 
recommended for these patients.

\section{FUTURE PERSPECTIVES}

Widespread use of ESD

ESD has higher en bloc resection rate and Ro resection rate than EMR. However, piecemeal EMR is still conducted in some areas. Widespread use of ESD is desired by overcoming its technical difficulties.

\section{Best treatment for circumferential lesions}

ESD is a minimally invasive treatment with high curative potential in patients with esophageal cancer. However, circumferential endoscopic resection can result in intractable stenosis, considerably reducing the patient's quality of life. Chemoradiotherapy is another option for circumferential lesions. A literature search failed to find any previous studies that described the specific survival rates of patients with circumferential lesions. Survival analyses and comparative studies of ESD and chemoradiotherapy for circumferential lesions are therefore required to determine the best treatment for circumferential lesions.

\section{Curability assessment for ( $p) M M$ cancer}

Assessing the curability of (p)MM cancer without vascular invasion is an issue in clinical practice. Previous studies examining the incidence of metastasis following endoscopic resection were conducted retrospectively, did not make it clear if the pathological evaluations were performed using immunostaining, and lacked thorough and long-term follow-up observations. Future prospective studies are anticipated to evaluate the metastasis rates in patients with (p)MM cancer based on detailed histological evaluations and intensive follow-up.

\section{Indication of additional treatment after ESD}

The indications for additional treatment are mainly determined based on the risk of metastasis and the patient's condition. Considering our aging society, additional treatment may not be indicated even in patients with a substantial risk of metastasis. However, detailed stratification of metastasis risk based on histologic findings is currently not possible, and further studies are needed to develop factors to determine detailed individual risks of metastasis.

\section{Conclusion}

An accurate preoperative diagnosis, appropriate indication, and adequate curability assessment based on the pathological diagnosis of resected specimens are important for effective ESD.

\section{DECLARATIONS}

\section{Acknowledgments}

I thank Susan Furness, PhD from Edanz (https://jp.edanz.com/ac) for editing a draft of this manuscript.

\section{Authors' contributions}

The author contributed solely to the article.

\section{Availability of data and materials}

Not applicable.

\section{Financial support and sponsorship}

None. 


\section{Conflicts of interest}

The author declared that there are no conflicts of interest.

\section{Ethical approval and consent to participate \\ Not applicable.}

\section{Consent for publication}

Not applicable.

\section{Copyright}

(c) The Author(s) 2021.

\section{REFERENCES}

1. Ferlay J, Colombet M, Soerjomataram I, et al. Estimating the global cancer incidence and mortality in 2018: GLOBOCAN sources and methods. Int J Cancer 2019;144:1941-53. DOI PubMed

2. Pouw RE, Heldoorn N, Alvarez Herrero L, et al. Do we still need EUS in the workup of patients with early esophageal neoplasia? Gastrointest Endosc 2011;73:662-8. DOI PubMed

3. Thosani N, Singh H, Kapadia A, et al. Diagnostic accuracy of EUS in differentiating mucosal versus submucosal invasion of superficial esophageal cancers: a systematic review and meta-analysis. Gastrointest Endosc 2012;75:242-53. DOI PubMed

4. Kitagawa Y, Uno T, Oyama T, et al. Esophageal cancer practice guidelines 2017 edited by the Japan esophageal society: part 2. Esophagus 2019;16:25-43. DOI PubMed PMC

5. Park CH, Yang DH, Kim JW, et al. Clinical practice guideline for endoscopic resection of early gastrointestinal cancer. Clin Endosc 2020;53:142-66. DOI PubMed PMC

6. Evans JA, Early DS, Chandraskhara V, et al; ASGE Standards of Practice Committee; American Society for Gastrointestinal Endoscopy. The role of endoscopy in the assessment and treatment of esophageal cancer. Gastrointest Endosc 2013;77:328-34. DOI PubMed

7. Othman MO, Lee JH, Wang K. Clinical practice update on the utility of endoscopic submucosal dissection in T1b esophageal cancer: expert review. Clin Gastroenterol Hepatol 2019;17:2161-6. DOI PubMed

8. Pimentel-Nunes P, Dinis-Ribeiro M, Ponchon T, et al. Endoscopic submucosal dissection: European Society of Gastrointestinal Endoscopy (ESGE) Guideline. Endoscopy 2015;47:829-54. DOI PubMed

9. Ajani JA, D'Amico TA, Bentrem DJ, et al. Esophageal and esophagogastric junction cancers, version 2.2019, NCCN Clinical Practice Guidelines in oncology. J Natl Compr Canc Netw 2019;17:855-83. DOI PubMed

10. Ishihara R, Matsuura N, Yano T, et al. ID: 3517386 usefulness of endoscopic ultrasonography in diagnosing cancer invasion depth of esophageal squamous cell carcinoma: a multicenter, prospective, single-arm, confirmatory trial (JCOG1604). Gastrointestinal Endoscopy 2021;93:AB236-7. DOI

11. Goda K, Tajiri H, Ikegami M, et al. Magnifying endoscopy with narrow band imaging for predicting the invasion depth of superficial esophageal squamous cell carcinoma. Dis Esophagus 2009;22:453-60. DOI PubMed

12. Mizumoto T, Hiyama T, Oka S, et al. Diagnosis of superficial esophageal squamous cell carcinoma invasion depth before endoscopic submucosal dissection. Dis Esophagus 2018:31. DOI PubMed

13. Ishihara R, Arima M, Iizuka T, et al; Japan Gastroenterological Endoscopy Society Guidelines Committee of ESD/EMR for Esophageal Cancer. Endoscopic submucosal dissection/endoscopic mucosal resection guidelines for esophageal cancer. Dig Endosc 2020;32:452-93. DOI PubMed

14. Oyama T, Inoue H, Arima M, et al. Prediction of the invasion depth of superficial squamous cell carcinoma based on microvessel morphology: magnifying endoscopic classification of the Japan Esophageal Society. Esophagus 2017;14:105-12. DOI PubMed PMC

15. Kim SJ, Kim GH, Lee MW, et al. New magnifying endoscopic classification for superficial esophageal squamous cell carcinoma. World J Gastroenterol 2017;23:4416-21. DOI PubMed PMC

16. Fujiwara J, Momma K, Tateishi Y. Endoscopic and pathological studies on type B2 blood vessels in estimation of invasion depth of superficial esophageal cancer. Stomach Intestine 2014;49:174-85. DOI

17. Dobashi A, Goda K, Kobayashi H. Clinical significance of type B1 vessels in the Japan esophageal society classification. Stomach Intestine 2014;49:153-63. DOI

18. Takeuchi M, Hashimoto S, Kobayashi M. Prospective study of the usefulness of type B2 vessel determined by the Japan esophageal society classification of magnified endoscopy for the diagnosis of superficial esophageal squamous cell carcinoma. Stomach Intestine 2014;49:164-72. DOI

19. Kadota T, Yano T, Kato T, et al. Prophylactic steroid administration for strictures after endoscopic resection of large superficial esophageal squamous cell carcinoma. Endosc Int Open 2016;4:E1267-74. DOI PubMed PMC

20. Hashimoto S, Kobayashi M, Takeuchi M, Sato Y, Narisawa R, Aoyagi Y. The efficacy of endoscopic triamcinolone injection for the prevention of esophageal stricture after endoscopic submucosal dissection. Gastrointest Endosc 2011;74:1389-93. DOI PubMed

21. Sato H, Inoue H, Kobayashi Y, et al. Control of severe strictures after circumferential endoscopic submucosal dissection for 
esophageal carcinoma: oral steroid therapy with balloon dilation or balloon dilation alone. Gastrointest Endosc 2013;78:250-7. DOI PubMed

22. Hanaoka N, Ishihara R, Uedo N, et al. Refractory strictures despite steroid injection after esophageal endoscopic resection. Endosc Int Open 2016;4:E354-9. DOI PubMed PMC

23. Takahashi H, Arimura Y, Okahara S, et al. A randomized controlled trial of endoscopic steroid injection for prophylaxis of esophageal stenoses after extensive endoscopic submucosal dissection. BMC Gastroenterol 2015;15:1. DOI PubMed PMC

24. Hashimoto S, Takeuchi M, Mizuno K. Prevention of stricture following semi-circular or circular esophageal ESD. Stomach Intestine 2013;48:1303-9. DOI

25. Yamaguchi N, Isomoto H, Fukuda H. Preventing stenosis after circumferential and semi-circumferential esophageal ESD -effect of oral steroid administration. Stomach Intestine 2013;48:1291-302. DOI

26. Miwata T, Oka S, Tanaka S, et al. Risk factors for esophageal stenosis after entire circumferential endoscopic submucosal dissection for superficial esophageal squamous cell carcinoma. Surg Endosc 2016;30:4049-56. DOI PubMed

27. Funakawa K, Uto H, Sasaki F, et al. Effect of endoscopic submucosal dissection for superficial esophageal neoplasms and risk factors for postoperative stricture. Medicine (Baltimore) 2015;94:e373. DOI PubMed PMC

28. Isomoto H, Yamaguchi N, Nakayama T, et al. Management of esophageal stricture after complete circular endoscopic submucosal dissection for superficial esophageal squamous cell carcinoma. BMC Gastroenterol 2011;11:46. DOI PubMed PMC

29. Kataoka M, Anzai S, Shirasaki T, et al. Efficacy of short period, low dose oral prednisolone for the prevention of stricture after circumferential endoscopic submucosal dissection (ESD) for esophageal cancer. Endosc Int Open 2015;3:E113-7. DOI PubMed PMC

30. Yamaguchi N, Isomoto H, Nakayama T, et al. Usefulness of oral prednisolone in the treatment of esophageal stricture after endoscopic submucosal dissection for superficial esophageal squamous cell carcinoma. Gastrointest Endosc 2011;73:1115-21. DOI PubMed

31. Yamashina T, Ishihara R, Uedo N, et al. Safety and curative ability of endoscopic submucosal dissection for superficial esophageal cancers at least $50 \mathrm{~mm}$ in diameter. Dig Endosc 2012;24:220-5. DOI PubMed

32. Matsueda K, Matsuura N, Kanesaka T, et al. Validity of endoscopic resection for clinically diagnosed T1a-MM/T1b-SM1 N0 M0 esophageal squamous cell carcinoma. Esophagus 2021;18:585-93. DOI PubMed

33. Akutsu Y, Kato K, Igaki H, et al. The prevalence of overall and initial lymph node metastases in clinical T1N0 thoracic esophageal cancer: from the results of JCOG0502, a prospective multicenter study. Ann Surg 2016;264:1009-15. DOI PubMed

34. Mitobe J, Ikegami M, Urashima M, Takahashi H, Goda K, Tajiri H. Clinicopathological investigation of lymph node metastasis predictors in superficial esophageal squamous cell carcinoma with a focus on evaluation of lympho-vascular invasion. Scand $J$ Gastroenterol 2013;48:1173-82. DOI PubMed

35. Yamashina T, Ishihara R, Nagai K, et al. Long-term outcome and metastatic risk after endoscopic resection of superficial esophageal squamous cell carcinoma. Am J Gastroenterol 2013;108:544-51. DOI PubMed

36. Eguchi T, Nakanishi Y, Shimoda T, et al. Histopathological criteria for additional treatment after endoscopic mucosal resection for esophageal cancer: analysis of 464 surgically resected cases. Mod Pathol 2006;19:475-80. DOI PubMed

37. Hatta W, Koike T, Takahashi S, et al; Tohoku GI Endoscopy Group. Risk of metastatic recurrence after endoscopic resection for esophageal squamous cell carcinoma invading into the muscularis mucosa or submucosa: a multicenter retrospective study. $J$ Gastroenterol 2021. DOI PubMed 